\title{
La cuantificación difuminada en la cuenta anual de una Oficina de Transferencia Tecnológica: ¿retórica o estilo?
}

\author{
Germán Varas ${ }^{1}$ \\ Pontificia Universidad Católica de Chile, Chile \\ Université de Rennes 1, Francia \\ Omar Sabaj ${ }^{2}$ \\ Universidad de La Serena, Chile
}

\begin{abstract}
Resumen
El uso de la cuantificación es un recurso gramatical y discursivo especialmente importante en el género de las cuentas públicas. En ellas, la cuantificación se utiliza para poner en valor los logros obtenidos por una institución. En el caso de una cuenta pública de una Oficina de Transferencia Tecnológica, estos logros corresponden a firmas de contratos, patentes, proyectos adjudicados o fondos de financiamiento, entre otros indicadores conmensurables de productividad. La cuantificación en estos textos puede presentarse de manera difuminada o desdibujada, por ejemplo, nuestra oficina revisó más de 200 emprendimientos. En el siguiente estudio, describimos, usando métodos mixtos (estudio de casos con fases
\end{abstract}

\footnotetext{
1 Para correspondencia, dirigirse a: Germán Varas (german.varas@univ-rennes1.fr), Av. Vicuña Mackenna 4860, Macul, Santiago.

2 Para correspondencia, dirigirse a: Omar Sabaj (omarsabaj@userena.cl), Raúl Bitrán 1305, La Serena.
} 
cuantitativas), el funcionamiento de la cuantificación difuminada en el discurso de una cuenta pública llevada a cabo por una Oficina de Transferencia y Licenciamiento. Los resultados muestran que el uso de la cuantificación difuminada representa casi la mitad de las veces que se cuantifica. Existen tres tipos semánticos de cuantificadores difusos, aquellos que se aproximan a un vector (aproximantes), aquellos que pasan un vector (traspasantes) y aquellos que difuminan un número (difuminantes). Estos últimos recursos son los más típicos para cuantificar de manera difusa. Asimismo, presentamos evidencia que favorece una explicación estilística, por sobre una retórica, del uso de la cuantificación difuminada en el discurso de la cuenta pública analizada.

Palabras clave: cuenta pública, cuantificación, difuminación, transferencia tecnológica, análisis del discurso.

\title{
FUZZY QUANTIFICATIONS IN THE ANNUAL ACCOUNT OF A
} Technology Transfer OfFice: Rhetoric OR STYle?

\begin{abstract}
Quantification is a grammatical and discursive resource especially important in the public account genre. In this type of texts, quantification is used to valorize the achievements of an institution. In the public account of a Technology Transfer Office, these achievements may refer to contracts, patents, awarded projects or funds, among other measurable indicators of productivity. Quantification in public accounts can be potentially expressed in a fuzzy way, for example, "our office reviewed more than 200 ventures". Using mixed methods (a case study with quantitative phases), we describe how fuzzy quantities operate in the public account rendered by a Chilean Technology Transfer Office. Our analysis showed that fuzzy quantifications correspond to almost $50 \%$ of the total quantifications. Three semantic types of fuzzy quantifiers were found: those approaching a vector (approximants), those exceeding a vector ('overreachers') and those blurring a number (diffusers). The latter was the most frequent resource for fuzzy quantifications. Furthermore, we provide evidence that would favor a stylistic explanation over a rhetorical one regarding the fuzzy quantification in the discourse of the analyzed public account.
\end{abstract}

Keywords: Public account, quantification, fuzziness, technology transfer, discourse analysis.

Recibido: 05/11/19 Aceptado: $11 / 03 / 20$ 


\section{INTRODUCCIÓN ${ }^{3}$}

La cuantificación es un recurso expresivo muy frecuente en el uso de la lengua, especialmente relevante en el género discursivo de las cuentas públicas. En este tipo de instancias, estos géneros tienen como propósito mostrar, mediante un discurso público oral, los logros obtenidos por una institución respecto de su desempeño en el periodo de un año.

Una de estas instituciones son las Oficinas de Transferencia y Licenciamiento (OTL), organismos que tienen por objetivo, al alero de una universidad, fomentar y coordinar la transferencia tecnológica. La "transferencia tecnológica" es el proceso de transformar el conocimiento científico en una tecnología que impacte en la sociedad. Entre sus funciones, las OTL gestionan la propiedad intelectual de la tecnología desarrollada por el académico/a, crean alianzas estratégicas entre la universidad y la industria $(\mathrm{I}+\mathrm{D})$ y fijan los términos comerciales entre las partes (investigadores, universidad, capitalistas de riesgo, y clientes interesados).

Un aspecto clave del discurso de la transferencia tecnológica es la valorización, mediante cuantificación, de los logros que consiguen los diferentes actores a lo largo del proceso, por ejemplo, firmas de contratos entre universidad y empresas o adquisición de fondos (públicos o privados) para el financiamiento de proyectos. Una de las instancias o momentos de valuación (Berthoin, Hutter \& Stark 2015) más importantes en las que se materializan los logros conseguidos por las oficinas de transferencia tecnológica, así como el de otras instituciones, es el de la cuenta pública. Este género se caracteriza, entre otros aspectos, por la inclusión estratégica de cuantificaciones que, como una forma de provocar reacciones (Espeland $\&$ Stevens 1998), tienen el potencial de crear valor, por ejemplo, aludiendo a cuán capaces/hábiles, benevolentes u honestos/éticos (en términos de Mayer, Davis \& Schoorman 1995) son los actores que forman parte del proceso de transferencia.

La cuantificación de tipo conmensurable (Espeland\& Stevens 1998) puede plasmarse de manera precisa o difuminada en el discurso, lo que se manifiesta en la oposición existente entre los siguientes enunciados: 'se firmaron 22 acuerdos' en comparación con 'se solicitaron casi 60 solicitudes

\footnotetext{
3 Esta investigación se realizó gracias al financiamiento del Programa del Fondo Nacional de Desarrollo Científico y Tecnológico (Fondecyt), Proyecto $\mathrm{N}^{\circ} 1170133$ y a los aportes del Centro de Investigación en Economía y Administración (CREM) de la Universidad de Rennes 1, Francia.
} 
de patentes'. Al reproducir una cuenta pública (a menudo planificada cuidadosamente en el "backstage" (Wodak 2015; Scollon and Scollon 2003 que conforma la transferencia tecnológica en tanto práctica social), el hablante opta por formas particulares de cuantificar.

Aunque este fenómeno ha sido descrito para otros géneros, la cuantificación difuminada no ha sido abordada en el estudio de las cuentas públicas. En este contexto, el objetivo de este trabajo fue describir el uso de las cuantificaciones difuminadas en una cuenta anual de una cuenta pública de una OTL.

En la primera parte de este artículo se hace una referencia general al dominio de la transferencia tecnológica, y a la cuantificación y la difuminación, tanto desde un punto de vista gramatical como discursivo. A continuación, mostramos la forma de recolección y análisis de los datos, los resultados obtenidos y su discusión. Finalmente, se ofrecen algunas conclusiones generales.

\section{LA TRANSFERENCIA TECNOLÓGICA: ACTORES Y FUNCIONES}

La transferencia tecnológica ha sido definida por la Corporación de Fomento de la Producción de Chile, CORFO, como "el proceso en el cual se pone en valor las capacidades de investigación de los centros desarrolladores de conocimiento al incorporar los resultados al quehacer del país" (CORFO 2016, p. x). Desde un punto de vista sociológico, la transferencia tecnológica es una actividad de producción económica que requiere altos niveles de cooperación e interdependencia entre sus participantes (Pina-Stranger \& Lazega 2011). Entre los actores e intereses que se movilizan en este proceso, se encuentran, por ejemplo, un académico, cuya línea de investigación tiene el potencial de convertirse en un producto transable en el mercado; una entidad privada, interesada en participar como capitalista de riesgo a cambio de beneficios; un comprador de la tecnología, que puede ser público o privado. Uno de los actores más importantes en el proceso de transferencia son las Oficinas de Transferencia Tecnológica y Licenciamiento, las cuales funcionan como agentes coordinadores de las interdependencias de los diversos actores.

Las Oficinas de Transferencia y Licenciamiento (OTL) son entidades que funcionan al alero de una universidad y se encargan de gestionar o, más bien mediar (Doganova 2013), la transformación de una investigación científica en un producto comercializable que satisfaga las demandas del 
mercado. Estas oficinas usualmente operan de manera interdependiente, utilizando diversos mecanismos de difusión y comunicación con terceras partes, como académicos, consorcios, capitalistas de riesgo, incubadoras, entre otros actores.

Los participantes de las oficinas de transferencia tecnológica, la mayoría especialistas con formación profesional en negocios, propiedad intelectual y la industria (CORFO 2016), conforman lo que Heckscher y Adler (2007) han denominado "comunidad colaborativa". Según estos autores, estas comunidades se caracterizan por tres atributos: a) mantienen estructuras sociales que apoyan procesos de administración interdependientes a través de vías formales e informales, b) reconcilian las múltiples identidades en conflicto, construyendo un sentido de unidad a partir de vínculos e interacciones apropiadas y, c) coordinan su actividad, basándose en el valor de la confianza, a través de compromisos comunes (por ejemplo, lograr la mayor cantidad de solicitudes de patentes, acuerdos tecnológicos y, por supuesto, ganancias).

En el caso de Chile, en particular, las Oficinas de Transferencia y Licenciamiento fomentan constantemente la transferencia entre los académicos y protegen las tecnologías mediante patentes; junto con lo anterior, las OTL, una vez que patentan una invención, obtienen retornos por licencias y acuerdos entre la universidad y la industria $(\mathrm{I}+\mathrm{D})$. La universidad, por su parte, además de recibir parte de las ganancias por patentamiento, es reconocida por el organismo regulador de la Propiedad Intelectual en Chile (INAPI) y, en consecuencia, adquiere prestigio a nivel nacional e internacional. El funcionamiento exitoso de este proceso de interdependencias o de compromiso común (Heckscher \& Adler 2007) en torno a las patentes requiere "narrativas discursivas" (Law 1994; Doolin 2005) o "guiones" (Pina-Stranger 2017) que legitiman sus acciones y resultados particulares (Dunford \& Jones 2000). Un instrumento para este propósito, como veremos, son las denominadas cuentas públicas.

\section{LA CUENTA PÚBLICA COMO GÉNERO DISCURSIVO}

Si bien el nombre del género hace referencia explícita a la actividad de contar, no existe consenso sobre lo que caracteriza una "cuenta pública". Sinclair (1995) se refiere al aspecto "camaleónico" de estos discursos, dado que pueden adoptar diversos significados según los diferentes contextos. En 
términos aristotélicos, la cuenta pública corresponde a un género epideíctico, esto es, de carácter ceremonial y/o demostrativo.

En estos discursos, los representantes de una institución muestran, de manera estratégica y articulada, los logros alcanzados. Estos discursos son estratégicos en tanto existe una asimetría de conocimiento entre los participantes. Así, mientras el orador tiene acceso a todos los datos que necesita para realizar su cuenta, la audiencia no sabe nada específico de manera previa, por lo cual el orador tiene la posibilidad de recontextualizar su discurso (van Leeuwen 2008), esto es, reorganizar, agregar, eliminar, legitimar, enfatizar o matizar la información que se presenta.

Las cuentas públicas, si consideramos el marco de referencia de Fairclough (2003), son prácticas discursivas que, al estar insertas en una práctica sociocultural (i.e., la transferencia tecnológica), responden a restricciones institucionales, sociales y situacionales. Similarmente, en términos de van Leeuwen (2008), las cuentas públicas podrían plantearse como "formas socialmente reguladas de hacer cosas" (p. 7). En este sentido, se pueden identificar diferentes características, por ejemplo, combinan siempre diversos modos semióticos, ya que suelen interactuar la oralidad con la escritura, el sonido, la imagen, los gestos y las expresiones faciales.

Además, cada institución reporta en su cuenta pública (o lo que podríamos llamar "práctica de cálculo", según Miller 2001) las entidades que se consideran relevantes de trasparentar en dicho evento social. Por ejemplo, en las cuentas públicas de un país, se espera que los presidentes expliciten públicamente el avance de los programas gubernamentales; la cuenta pública de una empresa, en cambio, utilizará otras entidades para demostrar su desempeño institucional, como las ventas y las inversiones, actividad que incluso contribuye al "sentido de unidad". (Heckscher \& Adler 2007)

Las Oficinas de Transferencia Tecnológica suelen aludir a materialidades para mostrar su desempeño, por ejemplo, el número de solicitudes y adjudicación de patentes, la cantidad de contratos o acuerdos de I+D firmados, el número de proyectos adjudicados, entre otros elementos. Estas materialidades (Latour 1991, 2007) cumplen un rol decisivo en el "ordenamiento" de una organización, dado que determinan el comportamiento de los individuos que la componen (Law 1994); en otros términos, las materialidades permiten que las instituciones que realizan transferencia tecnológica adquieran y promuevan sus propias identidades (por ejemplo, "las que más transfieren", "las más comprometidas con la innovación en el país", "las más productivas", etc.). 


\section{LA CUANTIFICACIÓN Y SU DIFUMINACIÓN COMO RECURSO SEMÁNTICO, GRAMATICAL Y DISCURSIVO}

La cuantificación, como categoría semántica, es la expresión de los significados asociados a las cantidades y grados. Como todo significado semántico es transversal, las cuantificaciones pueden materializarse léxica, gramatical y sintácticamente en distintas categorías o elementos cuantificadores (Kempson \& Cormack 1981). Los cuantificadores prototípicos son los adjetivos numerales cardinales que determinan (i.e., determinantes cuantificadores) y preceden al sustantivo como en 'diez contratos', pero también existen sustantivos cuantificativos como 'una docena' o 'un montón'.

Los elementos cuantificadores pueden, a su vez, ser afectados por otros elementos, entre otros, locuciones preposicionales que, si bien cuantifican, no lo hacen de manera exacta como en 'más de diez contratos'. Es interesante notar que el tipo gramatical del elemento cuantificador varía según la naturaleza semántica del elemento cuantificado (di Tullio 2010). Así, por ejemplo, si bien los sustantivos contables pueden ser cuantificados con los determinantes cuantificadores cardinales o con determinantes cuantificadores indefinidos ('tres libros', 'diversos libros'), los nombres incontables solo pueden ser afectados por elementos indeterminados ('mucha agua' versus '\#tres aguas', que es anómalo).

La noción de difuminación tiene una larga tradición en lingüística cognitiva (Talmy 2000). En una primera acepción, la noción se aplica a las categorías conceptuales, esto es, a las clases de conceptos. Así, por ejemplo, se habla de miembros prototípicos y periféricos de una clase. Mientras el zorzal es un pájaro prototípico, los pingüinos no lo son. En una segunda acepción (Lakoff 1972), la idea de difuminación dice relación con el uso de atenuadores ('poco frío'), enfatizadores (muy frío) o difuminadores ('algo frío') para expresar juicios. De esta manera, existe un vínculo entre el uso de los cuantificadores y su difuminación mediante el uso de distintos elementos gramaticales.

El funcionamiento de los intensificadores y de los atenuadores como recursos de difuminación ha recibido, en general, vasta atención, por ejemplo, como elemento clave en las narraciones (Labov 1972) y como fenómeno pragmático de cortesía (Albelda 2008), pero también considerando aspectos contextuales y culturales (Puga 1997). En el ámbito del discurso científico, en especial, este tema se ha desarrollado por más de tres décadas (Coates 1983; Palmer 1990; Hyland 1996; Hood 2004; Martin \& White 2005; Hyland \& Jiang 2016; entre varios otros). 
Hyland (1996, 2000, 2008), por ejemplo, uno de los principales investigadores que ha analizado estos recursos en el género artículo académico, establece que los intensificadores y atenuadores permiten definir el estatus profesional de los individuos y representan un medio retórico crítico para conseguir la aceptación de las reivindicaciones epistémicas entre los pares. Estos dispositivos de difuminación, señala el autor, ayudan a los académicos a conseguir aceptación en su "práctica social", equilibrando convicción con precaución y modestia con asertividad.

Los intensificadores como "claramente", "obviamente", y "por supuesto" permiten a los hablantes expresar convicción y remarcar su compromiso y solidaridad con su audiencia. Los atenuadores, en tanto recurso de difuminación, como "probablemente", "podría" y "algo" (kind of), permiten a los científicos insertarse discursivamente en un mundo donde la precaución y la precisión son esenciales. (Hyland 1996)

En la teoría sistémico-funcional, la atenuación y la intensificación en tanto recurso de difuminación son considerados como elementos semióticos que permiten graduar significados evaluativos, como apreciaciones, afectos y juicios (Martin \& White 2005). En términos generales, Martin y White (2005) establecen en su modelo de Valoración que el sistema de GRADUACIÓN opera en dos ejes de escalabilidad: a) aquel que gradúa según intensificaciones o cuantificaciones (denominado Fuerza); y b) aquel que gradúa de acuerdo con la prototipicidad y la precisión con que se dibujan los límites de una categoría (denominado Foco).

En cuanto al eje de Fuerza, las intensificaciones tienen la particularidad de que pueden realizarse mediante un ítem aislado (muy interesante) o un lexema que fusiona gradualidad con valoración (feliz, contento, extasiado). Las cuantificaciones, por su parte, tienen el potencial, señalan Martin y White (2005), de graduar a través de estimaciones imprecisas de cantidades (pocos, muchos), estimaciones imprecisas de masa o presencia (pequeño, grande; delgado, grueso; ligero, pesado; oscuro, brilloso) y estimaciones imprecisas con respecto a una extensión en el tiempo y el espacio, ahora medidos en términos de proximidad (cercano, lejano; reciente, antiguo) o distribución (por ejemplo, demasiado largo, en el corto plazo; ampliamente difundido). En cuanto al eje de foco, las gradaciones por prototipicidad operan en la medida en que los fenómenos son escalados mediante referencia al grado al que se corresponden con un supuesto núcleo o instancia ejemplar de alguna categoría semántica (es algo inconveniente), es decir, dependiendo de su grado de prototipicidad (idea similar a la planteada por Rosch 1975). 
Desde nuestra perspectiva, las cuantificaciones mediante estimaciones imprecisas, como las descritas anteriormente, corresponden a recursos de desdibujamiento; de esta forma, tendrían mayor relación con el eje de foco que con el de Fuerza. Por lo tanto, creemos que el eje de Foco de Martin y White (2005) no debería limitarse a una semántica de prototipicidad, en términos de la pertenencia a una categoría, sino que debería considerar también su potencial semiótico como mecanismo para graduar las cantidades de manera difusa.

El uso de la cuantificación difuminada tiene, desde un punto discursivo, motivaciones retóricas estratégicas. Dubois (1987) denominó a este fenómeno la "retórica de la imprecisión" (o la vaguedad, en términos de Channell 1990). En su estudio, Dubois (1987) analizó la imprecisión de las expresiones numéricas que aparecían en las presentaciones de los científicos en el marco de un congreso de Biomedicina. Entre los resultados, encontró que las imprecisiones se realizaban, por un lado, a través de cifras redondeadas, rangos, fracciones y múltiples comunes; y por otro, mediante atenuadores. Entre los atenuadores, identificó casos como "un poco" ( $a$ little), "en torno a" (in that around), "cercano a" (close to), "no más de" (no much longer than), "más/menos" (plus/minus), "cercano a" (near-ly), "algo así como" (something like), "más o menos" (or so), "aproximadamente" (approximately), "o más" (or more), "casi" (almost) y "en el orden de" (in the order of).

Al poner estos resultados en contexto, Dubois (1987) encontró que los científicos difuminaban las cifras cuando: a) los resultados eran muy preliminares; b) se experimentaba con cantidades muy pequeñas de animales; c) se empleaban técnicas o dispositivos potencialmente cuestionables y d) cuando había dificultad para medir a todos los miembros de una determinada clase. De esta forma, la imprecisión, concluye Dubois (1987), permite a los científicos realzar o magnificar aquellas otras cantidades que realmente consideran importantes dentro de sus investigaciones.

La cuantificación difuminada puede ser, en el contexto de una cuenta pública, una estrategia retórica que permite al orador seleccionar y enfatizar la información que él estima conveniente. Estas maniobras discursivas son posibles, si y solo si, la audiencia no tiene acceso al número real al cual se hace referencia. En este sentido, la retórica de la imprecisión que se manifiesta en las cantidades difuminadas exige asimetría epistémica entre los participantes del discurso (es decir, un principio de autoridad, en términos de Espeland y Stevens 1998). 


\section{MÉTODOS}

Esta investigación corresponde a un estudio de casos de un fenómeno particular, a saber, la cuantificación difuminada en una cuenta pública de una Oficina de Transferencia Tecnológica. El estudio tiene un alcance descriptivo y exploratorio. El enfoque es mixto, en tanto se utilizan estadísticas descriptivas para realizar un análisis cualitativo de categorías provenientes de la gramática, de la semántica y del análisis del discurso.

\subsection{LOS DATOS: RECOLECCIÓN Y ÉTICA}

Los datos primarios y crudos de esta investigación corresponden a 66 expresiones de cuantificación que aparecieron en el discurso de la Cuenta Pública emitida por el director de la Dirección de Transferencia y Desarrollo de la Pontificia Universidad Católica de Chile el 15 de mayo de 2018. Esta cuenta se realizó en el marco de la celebración del "Día de la transferencia tecnológica y propiedad intelectual UC 2018".

Aun cuando se trató de información pública, para la presente investigación se contó con la autorización del propio director de la Oficina de Transferencia y Licenciamiento (OTL) para registrar y, posteriormente, analizar el discurso del evento. El análisis específico se realizó sobre la transcripción literal de un total de 4.531 palabras, proporcionada por la dirección de la OTL.

Estos datos primarios fueron complementados con otras fuentes de información (datos secundarios), la mayoría de carácter público, como el audio y el video del evento, la presentación que acompañó a la cuenta, el informe oficial asociado a la cuenta (ver aquí) e información en sitios específicos (Inapi, UC, Corfo). Toda esta información fue utilizada, específicamente, para determinar una de las categorías analizadas (la de los números reales), las cuales explicamos a continuación.

\subsection{Procedimientos de ANÁLisis}

De los datos primarios se extrajeron todas las expresiones de cuantificación y se dispusieron con una ventana de 6 elementos de cotexto a cada lado en la primera columna de una planilla de cálculos. El segmento que expresaba la cuantificación se marcó en rojo y se clasificó en las siguientes columnas, según cada una de las seis categorías que aparecen listadas, definidas y ejemplificadas en la Tabla 1. 
Tabla 1: Definición operativa y ejemplos de las categorías de análisis

\begin{tabular}{|c|c|c|}
\hline Categoría & Definición & Ejemplo \\
\hline $\begin{array}{l}\text { El tipo de } \\
\text { cuantificación }\end{array}$ & $\begin{array}{l}\text { Desde un punto de vista } \\
\text { semántico, la cuantificación } \\
\text { puede manifestarse de } \\
\text { manera precisa o difusa }\end{array}$ & $\begin{array}{l}\text { Firmamos tres acuerdos } \\
\text { Firmamos más de tres acuerdos }\end{array}$ \\
\hline \begin{tabular}{|l|} 
Tipos de \\
cuantificadores \\
difusos
\end{tabular} & \begin{tabular}{|l} 
Los cuantificadores \\
difusos pueden clasificarse \\
en aproximantes (se \\
acercan a un vector), \\
traspasantes (traspasan un \\
vector) y cuantificadores \\
indeterminados (que están \\
cerca al vector, pero no se \\
sabe en qué dirección)
\end{tabular} & $\begin{array}{l}\text { Firmamos casi } 5 \text { acuerdos } \\
\text { Firmamos más de tres acuerdos } \\
\text { Firmamos alrededor } \\
\text { de } 100 \text { acuerdos }\end{array}$ \\
\hline \multirow{7}{*}{$\begin{array}{l}\text { Tipo de } \\
\text { elemento } \\
\text { gramatical }\end{array}$} & $\begin{array}{l}\text { Se distinguieron siete tipos } \\
\text { de categorías gramaticales: }\end{array}$ & \\
\hline & Adverbio & $\begin{array}{l}\text { Contempló la creación } \\
\text { de casi } 60 \text { viviendas }\end{array}$ \\
\hline & $\begin{array}{l}\text { Determinantes } \\
\text { cuantificadores cardinales }\end{array}$ & Se firmaron $\mathbf{4 2}$ acuerdos \\
\hline & $\begin{array}{l}\text { Determinantes } \\
\text { cuantificadores porcentuales }\end{array}$ & $\begin{array}{l}\text { Se perdió } \mathbf{1 2 , 5 \%} \text { de la } \\
\text { producción de vino }\end{array}$ \\
\hline & Determinantes indefinidos & $\begin{array}{l}\text { La DTD implementa } \\
\text { diversas estrategias }\end{array}$ \\
\hline & Locución preposicional & Más de 260 registros de PI \\
\hline & Pronombre cuantificador & 4 de ellas, eran patentes... \\
\hline \multirow{4}{*}{\begin{tabular}{|l|} 
El alcance \\
sintáctico de la \\
cuantificación
\end{tabular}} & Sujeto & $\begin{array}{l}41 \% \text { de estas licencias } \\
\text { fueron firmadas }\end{array}$ \\
\hline & Complemento directo & Se firmaron 42 acuerdos \\
\hline & $\begin{array}{l}\text { Término de un sintagma } \\
\text { preposicional }\end{array}$ & $\begin{array}{l}\text { Contaba con } 117 \\
\text { patentes concedidas }\end{array}$ \\
\hline & Adjunto & $\begin{array}{l}\text {.., destacando sus más de } \\
\text { diez años de estudios }\end{array}$ \\
\hline
\end{tabular}




\begin{tabular}{|l|l|l|}
\hline $\begin{array}{l}\text { Los números } \\
\text { declarados }\end{array}$ & $\begin{array}{l}\text { La expresión de la } \\
\text { cuantificación tal cual como } \\
\text { aparece en el discurso }\end{array}$ & "Más de 10 años" \\
\hline $\begin{array}{l}\text { Los números } \\
\text { reales }\end{array}$ & $\begin{array}{l}\text { La cantidad real a la que } \\
\text { el número declarado } \\
\text { hace referencia }\end{array}$ & $\mathbf{1 6}$ años \\
\hline
\end{tabular}

Para determinar cuán difuminados estaban "los números declarados", estos se contrastaron con los "números reales", los cuales fueron rastreados utilizando los datos secundarios antes descritos. La disponibilidad de esta información no fue total. Más concretamente, solo pudimos recuperar 13 números reales de las 32 instancias de cuantificación difuminada aparecidas (los números declarados) en el discurso.

Para garantizar la credibilidad y la fiabilidad del análisis realizado se utilizaron tres procedimientos. Primero, la identificación y definición inicial de las categorías se realizó en conjunto por ambos investigadores a partir de un análisis inductivo. Estas categorías se definieron luego formalmente, utilizando los criterios de la Tabla 1. El segundo procedimiento consistió en el análisis independiente de los datos por cada uno de los investigadores y, posteriormente, la resolución de discrepancias. Dado que las categorías estaban definidas de manera clara y formal, los desacuerdos en la clasificación fueron mínimos (ver comentarios sobre casos especiales en la Tabla 4). Por último, estos casos fueron resueltos a través de consulta a dos expertos en gramática, quienes además revisaron y ratificaron la aplicación de cada categoría a cada una de las instancias de aparición. Al aplicar cada una de las categorías de la Tabla 1 a los 66 casos originales obtuvimos una hoja de cálculos con 343 datos, desde los cuales se obtuvieron los resultados.

\section{RESULTADOS Y DISCUSIÓN}

Revisaremos primero, a modo de antecedente para la exposición de los resultados específicos sobre la cuantificación difuminada, los resultados referentes a la cuantificación exacta.

Treinta cuatro $(52 \%)$ de las 66 instancias de cuantificación se expresan de manera exacta. Casi la totalidad de las cuantificaciones exactas, el $88 \%$ (30/34) se expresa utilizando determinantes cuantificadores cardinales (1) y solo un $12 \%(4 / 34)$ se realizan con pronombres cuantificadores (2). Desde 
un punto de vista gramatical, entonces, la variedad de recursos gramaticales para expresar la cuantificación exacta es baja:

(1) El 2017 presentamos 58 solicitudes de patentes

(2) 13 de diseños industriales

Los elementos cuantificados de manera exacta aparecen en la Tabla 2:

Tabla 2: Listado de frecuencias asociadas a la cuantificación exacta en el discurso de una cuenta pública de una Oficina de Transferencia Tecnológica y Licenciamiento.

\begin{tabular}{|l|c|}
\hline Elementos cuantificados de manera exacta & $\mathbf{N}^{\circ}$ \\
\hline acuerdos & 6 \\
\hline patentes & 6 \\
\hline solicitudes de patentes & 6 \\
\hline obras & 4 \\
\hline licencias & 2 \\
\hline tecnologías & 2 \\
\hline barrios & 1 \\
\hline centros científicos & 1 \\
\hline charlas & 1 \\
\hline emprendimientos & 1 \\
\hline jardines infantiles & 1 \\
\hline pisos & 1 \\
\hline proyectos I+D & 1 \\
\hline universidades & 1 \\
\hline TOTAL & $\mathbf{3 4}$ \\
\hline
\end{tabular}

Si nos fijamos en los resultados, podemos observar que las materialidades cuantificadas de manera exacta más recurrentes (Latour 1991 y 2007) son relevantes ya que, desde un punto de vista social, estas entidades determinan las prácticas y todas las actividades en torno a la transferencia tecnológica: los acuerdos alcanzados, las solicitudes de patentes, las obras, las licencias y las tecnologías son, en estos términos, aquellas entidades que se utilizan para mostrar los logros y las capacidades de la institución que realiza la cuenta pública.

La función sintáctica de los elementos cuantificados de manera exacta se muestra en la Tabla 3. 
Tabla 3: Alcance sintáctico de los elementos cuantificadores exactos

\begin{tabular}{|l|c|c|l|}
\hline $\begin{array}{l}\text { Alcance del elemento } \\
\text { cuantificador }\end{array}$ & $\mathbf{N}$ & $\mathbf{\%}$ & Ejemplos \\
\hline $\begin{array}{l}\text { Término de sintagma } \\
\text { preposicional }\end{array}$ & 14 & 41 & $\begin{array}{l}\text { (3) Actualmente contamos con 22 } \\
\text { alianzas de I+D }\end{array}$ \\
\hline Complemento directo & 12 & 35 & $\begin{array}{l}\text { (4) El } 2017 \text { presentamos 58 solicitudes } \\
\text { de patentes }\end{array}$ \\
\hline Sujeto & 8 & 24 & $\begin{array}{l}\text { (5) 5 obras desarrolladas por } \\
\text { académicos de la Facultad fueron ... }\end{array}$ \\
\hline Total & $\mathbf{3 4}$ & $\mathbf{1 0 0}$ & \\
\hline
\end{tabular}

Es interesante notar que las cuantificaciones exactas aparecen frecuentemente asociadas a términos de sintagmas de verbos que tienen régimen preposicional (di Tullio 2007) como 'contar con 22 alianzas' (3), 'asociarse a 6 universidades'; o bien a complementos de sustantivos nominalizados como 'la creación de 8 tecnologías'. También es común que las entidades cuantificadas ocupen la posición del objeto como en (4) y de manera menos frecuente que aparezcan asociadas al sujeto de la oración como en (5).

En términos generales, podemos establecer que la cuantificación exacta representa apenas un poco más de la mitad de las expresiones cuantificadas $(52 \%)$ de la cuenta pública analizada. La mayoría de estos elementos son determinantes cuantificadores cardinales, que cumplen la función sintáctica de términos de sintagmas preposicionales que afectan específicamente a los siguientes sustantivos: acuerdos, patentes, solicitudes de patentes, obras.

La expresión de la cuantificación difuminada, que es el foco de esta investigación, representa casi la mitad de las instancias de cuantificación que se expresan en la cuenta pública analizada, a saber, treinta y dos de las 66 instancias (48\%). La cuantificación difuminada, por tanto, representa una estrategia muy frecuente en este tipo de textos y su probabilidad de aparición cada 100 palabras $(0,70)$ es muy parecida a la de la cuantificación exacta $(0,75)$. 
Tabla 4: Frecuencia, distribución, ejemplos y casos especiales

\begin{tabular}{|c|c|c|c|}
\hline $\begin{array}{l}\begin{array}{l}\text { Tipo de } \\
\text { difuminación }\end{array} \\
\end{array}$ & $\mathbf{N}^{\circ}$ & $\%$ & Ejemplos \\
\hline \multirow{10}{*}{ Difuminante } & \multirow{10}{*}{20} & \multirow{10}{*}{63} & $\begin{array}{l}\text { (6) ... a través de diversos tipos de acuerdos } \\
\text { (7) Llevamos varios años reconociendo } \\
\text { (8) ....integraron diversos comités de validación } \\
\text { (9) ...es necesario cumplir ciertas etapas previas } \\
\text { (10) Hace pocas semanas el Instituto } \\
\text { Nacional de Propiedad Industrial, INAPI... } \\
\text { (11) ....alrededor de } 100 \text { millones ... }\end{array}$ \\
\hline & & & $\begin{array}{l}\text { Casos especiales } 1 \\
\text { (12) ...41\% de estas licencias fueron } \\
\text { firmadas con Universidades }\end{array}$ \\
\hline & & & $\begin{array}{l}\text { (13) ... se perdió } 12,5 \% \text { de la producción } \\
\text { de vino de la zona afectada }\end{array}$ \\
\hline & & & (14) $\ldots 32 \%$ con empresas \\
\hline & & & (15) $\ldots 23 \%$ corresponden a licencias de descarga \\
\hline & & & (16) $\ldots .5 \%$ al sector público \\
\hline & & & (17) ...por US $\$ 3,3$ millones de fondos privados \\
\hline & & & $\begin{array}{l}\text { (18) ...y actualmente la empresa está } \\
\text { valorizada en US \$13 millones }\end{array}$ \\
\hline & & & $\begin{array}{l}\text { (19) ...comprometiendo fondos por } \\
\text { un total de USD } \$ 3 \text { millones. }\end{array}$ \\
\hline & & & $\begin{array}{l}\text { (20) Durante } 4 \text { años fue diseñado } \\
\text { interdisciplinariamente }\end{array}$ \\
\hline \multirow{6}{*}{ Traspasante } & \multirow{6}{*}{9} & \multirow{6}{*}{28} & $\begin{array}{l}\text { (21) ...y que durante el } 2017 \text { revisó } \\
\text { más de } 200 \text { emprendimientos }\end{array}$ \\
\hline & & & $\begin{array}{l}\text { (22) ... nuestra dirección ha gestionado más } \\
\text { de } 260 \text { registros de derechos de autor }\end{array}$ \\
\hline & & & $\begin{array}{l}\text { (23) ...la postulación de más } \\
\text { de } 240 \text { proyectos de } I+D\end{array}$ \\
\hline & & & Casos especiales 2 \\
\hline & & & $\begin{array}{l}\text { (24) ... una tasa de adjudicación } \\
\text { de más del } 25 \% \text {... }\end{array}$ \\
\hline & & & $\begin{array}{l}\text { (25) ... donde más del } 50 \% \text { de } \\
\text { ellos son extranjeros... }\end{array}$ \\
\hline
\end{tabular}




\begin{tabular}{|c|c|c|c|}
\hline Aproximante & 3 & 9 & $\begin{array}{l}\text { (27) ...UC tiene adjudicado para investigación } \\
\text { y desarrollo cerca de } 7 \text { millones de dólares } \\
\text { (28) ...lo que derivó en la presentación de casi } \mathbf{6 0} \\
\text { solicitudes patentes en diversos países del mundo } \\
\text { (29) ... contempló la creación de casi } \\
\mathbf{8 0 0} \text { viviendas para nuestro país. }\end{array}$ \\
\hline Totales & 32 & 100 & \\
\hline
\end{tabular}

Desde un punto de vista semántico, la cuantificación difuminada se cristaliza mayoritariamente en elementos difuminantes (20/32), seguida de significados traspasantes (9/32) y, de forma minoritaria, en elementos de cuantificación aproximante (3/32). Cada una de estas categorías, su frecuencia y distribución con los ejemplos y casos especiales aparecen en la Tabla 4.

La expresión gramatical de la cuantificación difuminada es mucho más variable que la cuantificación exacta. A excepción del caso (11), la utilización de elementos difuminantes se concreta en determinantes indefinidos (diversos, ciertos, varios pocos), y está asociada con elementos léxicos que no tiene relación con las materialidades específicas de la transferencia tecnológica, sino que dice relación con el proceso general de la transferencia tecnológica (tipos de acuerdos, años, comités, etapas previas).

Es interesante notar que, en esta primera categoría, el único elemento léxico que se cuantifica por medio de una locución preposicional (alrededor de) corresponde a una instancia relativa al financiamiento (11). Nada relativo al dinero es referido de forma exacta (ver Tabla 2), aunque así pareciera a primera vista, según lo que se puede establecer de lo que en la Tabla 4 denominamos casos especiales. Estos casos surgieron al realizar el análisis de algunos de los datos, cuya clasificación provocó dificultades que fueron luego resueltas.

El primero de los casos decía relación con la manera de clasificar (exacta o difuminada) la expresión de la cuantificación a través de porcentajes (12-16). Dado que la expresión de porcentajes es la expresión de la proporción entre dos cantidades (frecuencia relativa) se consideraron todos estos casos como instancias de cuantificación difuminada, ya que, en efecto, en el discurso los logros de la OTL se muestran como proporciones. En otros términos, las materialidades extralingüísticas a las que hace referencia una cuenta pública son entidades finitas exactas (no existe algo así como una patente y media) y no una proporción de otras cosas y, por esa razón, se trata de un caso especial de cuantificación difuminada, una retórica de la vaguedad, en términos de Dubois (1987). 
Una segunda observación, que derivó en esta categoría de casos especiales se produjo en el análisis de los casos de los ejemplos (17) al (20). El uso de una cantidad de millones con decimales, tal como el que aparece en el ejemplo (17), también muestra que se trata de una estrategia para evitar decir el monto exacto. Los casos (18 al 20) son aún más curiosos. En estos casos, podemos hablar de una suerte de redondeo pragmático. En efecto, se trata de una estrategia de difuminación más sutil que no tiene siquiera una marca discursiva, sino que requiere de la interpretación de la audiencia.

Estos casos son controversiales porque se presentan como exactos en el discurso, pero obviamente no hacen referencia a entidades bien delimitadas. Por ejemplo, difícilmente, en el caso de (18), la empresa está exactamente valorizada en US \$13 millones o que en (19) se haga referencia de manera precisa a US \$3 millones o que los 4 años en (20) correspondan a la verdad lógica que equivale a multiplicar 4 años por 365 días. Este tipo de interpretaciones ha sido señalado también por Soto (2017), quien sostiene que en estos casos prevalece nuestro conocimiento de mundo por sobre la relación entre el lenguaje y la realidad.

El segundo tipo semántico de cuantificación difuminante se realiza a través de elementos traspasantes, cuya expresión única es la locución preposicional "más de". Tal como en los casos anteriores (del 11 al 20), esta construcción se utiliza para cuantificar las entidades que organizan y determinan la actividad de transferir tecnología: emprendimientos, registros de derechos de autor, proyectos de I+D. Esta locución preposicional se utiliza, principalmente, para afectar a determinantes cuantificadores cardinales como en los casos (21 a 23). Existen, sin embargo, otras formas especiales de cuantificación difuminada traspasante, como las que aparecen en los ejemplos (24 y 25), las cuales, por conveniencia hemos denominado determinantes cuantificadores porcentuales. Dado que ya hemos establecido que la utilización de porcentajes es en sí una estrategia de difuminación, lo especial de estos casos radica en su doble difuminación a partir de la adición del elemento traspasante ( $25 \%$ ya es difuso, más del $\mathbf{2 5 \%}$ es aún más difuso).

Por último, el tercer tipo semántico de cuantificación difuminada, que denominamos aproximantes, es el menos frecuente. Estos elementos se expresan mediante locuciones preposicionales como "cerca de" y de adverbios como "casi" que afectan a entidades como millones de dólares, solicitudes de patentes y viviendas, entre otros elementos léxicos.

El uso de la cuantificación en el discurso de las cuentas públicas y la proporción de cuantificación exacta y difuminada puede explicarse, en primer término, por razones retórico-estratégicas, entre las cuales se encuentra el hecho de que existe una asimetría entre el conocimiento del orador y su audiencia. Esta estrategia, que denominaremos la explicación retórica, 
podría parafrasearse en los siguientes términos: dado que la audiencia no tiene acceso a la cifra exacta, el uso de la cuantificación difuminada por parte del orador es una manera de aumentar (estirar, alargar o prolongar) discursivamente en la mente del auditorio esa cifra que en la realidad corresponde a una entidad finita, un número entero que no corresponde desde un punto de vista matemático con aquel declarado.

Una explicación alternativa sería que el uso de la cuantificación difuminada es, más bien, una cuestión de variación en el estilo. El hablante, con el propósito de hacer un uso variado de formas de expresión, intercala formas exactas y difuminadas de cuantificación. Para probar qué explicación tiene más asidero a la luz de los datos, pasamos a describir los hallazgos en relación con dichas explicaciones (retóricas o de estilo).

Para probar la explicación de que el uso de la cuantificación difuminada responde a una cuestión retórica, se deberían cumplir ciertas condiciones: 1) Que la mayoría de las veces que se cuantifique de manera difuminada, la audiencia no conozca los números reales y que, 2) el orador estire, lo más posible, la distancia entre el número real (nr) y el declarado (nd). La estrategia de cuantificación difuminada ideal estaría basada en una lógica retórica total si se cumplieran siempre estas dos condiciones.

Para responder a estas preguntas, accedimos a los datos secundarios. De la observación del video introductorio y de la presentación que acompañó la cuenta pública pudimos ver si se cumplían o no las condiciones descritas. Estos resultados aparecen en la Tabla 5.

Tabla 5: Los números reales, los números difuminados y su uso estratégico

\begin{tabular}{|c|l|c|c|c|}
\hline $\mathbf{N}^{\circ}$ & Número declarado (nd) & $\begin{array}{c}\text { Número } \\
\text { real (nr) }\end{array}$ & $\begin{array}{c}\text { Conocido por } \\
\text { la audiencia }\end{array}$ & $\begin{array}{c}\text { Uso } \\
\text { estratégico }\end{array}$ \\
\hline 1 & Diversos & 22 & Sí & No \\
\hline 2 & $41 \%$ & 9 & Sí & Sí \\
\hline 3 & $32 \%$ & 7 & Sí & Sí \\
\hline 4 & más de 200 & 246 & Sí & No \\
\hline 5 & $23 \%$ & 5 & Sí & Sí \\
\hline 6 & $5 \%$ & 1 & Sí & Sí \\
\hline 7 & más de 260 & 272 & Sí & No \\
\hline 8 & más de 240 & 246 & Sí & No \\
\hline 9 & más de 6000 & $4800^{*}$ & No & Sí \\
\hline 10 & Cerca de 7 millones de dólares & 7 & Sí & No \\
\hline
\end{tabular}

\footnotetext{
${ }^{*}$ No es el monto total
} 


\begin{tabular}{|c|l|c|c|c|}
\hline 11 & más de 10 & 10 & No & Sí \\
\hline 12 & casi 60 & 58 & Sí & Sí \\
\hline 13 & casi 800 & 747 & Sí & Sí \\
\hline
\end{tabular}

En el 59\% (19 de los 32 casos) en los que apareció la cuantificación difuminada ni la audiencia ni los investigadores tuvieron acceso (al menos visualmente) al número real, lo que, en parte, justifica la explicación retórica, tal como se presenta en la condición 1, pero, dado que son cantidades en la práctica inaccesibles, no es posible probar la condición 2.

Ahora bien, si analizamos en detalle el $41 \%$ restante ( 13 de los 32 casos), sucede que en la mayoría de ellos (11 de 13), la audiencia tenía acceso al número real obtenido de la presentación multimodal que acompañó a la cuenta pública. En casi la mitad de estos casos (5 de 11), el orador no los utilizó de manera estratégica (casos 1-4-7-8-10), ya que, por así decirlo, no estiró en todo su potencial el número declarado. Este análisis detallado le resta fuerza a la explicación retórica.

Para analizar la segunda condición de la explicación retórica de la cuantificación difuminada, determinamos si realmente el número declarado suponía una diferencia clara con el real, o, si al menos, discursivamente era conveniente. Por ejemplo, en el caso 1 era mucho mejor decir el número real (22) que utilizar el cuantificador indeterminado (diversos). Los casos 2, 3, 5 y 6 son inversos, ya que, estratégicamente, la expresión de la cantidad en proporciones permite aumentar la percepción de la cantidad en frecuencias brutas. El caso 4 es otra muestra de que la cuantificación difuminada no siempre se usa de manera estratégica, ya que el número real era 246 y el número declarado, más de 200. En este caso habría sido más estratégico utilizar un elemento difuminador aproximante (casi 250). Lo mismo sucede en los casos 7 y 8 . En síntesis, solo en 8 de los trece casos el orador estira la diferencia entre el número real y el difuminado.

Por lo tanto, de todos los casos de cuantificación difuminada en que ni los investigadores ni la audiencia tuvieron acceso (13 de 32), solo dos cumplen con lo que podríamos denominar la explicación retórica ideal. En estos casos se cumplían, a la vez, las dos condiciones que sustentan la explicación retórica, pero que, tal como hemos visto, son muy poco frecuentes.

Para probar la explicación estilística de la cuantificación, tomamos de la planilla de cálculos las celdas en los que aparecía la clasificación realizada, según si la cuantificación fuera difusa o exacta. Estos datos los traspasamos a un archivo de texto plano (.txt) y luego los cargamos en el software Antconc v.3.5.8 (Anthony, 2019), con el cual es posible obtener patrones o n-gramas y se puede observar cómo se distribuyen las frecuencias de las palabras según el 
número de veces que se repiten en un texto (Matsuda, Sadowsky, Matsuda \& Sabaj, 2012). Así, si las secuencias son poco variables, es decir, hay muchas secuencias de una misma categoría (exacta-exacta-exacta... o difusa-difusadifusa), la explicación estilística perdería sustento. En cambio, si lo que es más abundante son secuencias de categorías combinadas, con patrones identificables, diremos que el recurso de la cuantificación difuminada tiene una explicación basada en la variación de los recursos de la cuantificación, esto es, lo que hemos denominado la explicación estilística.

En la Figura 1 y 2 y en las Tablas 6 y 7 , se muestran los datos para probar la explicación estilística.

Figura 1. Concordance Plot exacta/difusa


La Figura 1 muestra la aparición a través del texto de las cuantificaciones exactas en negro y de las cuantificaciones difusas en rojo. Tal como se aprecia, la utilización de los recursos de cuantificación es evidentemente complementaria. Solo al final del texto existe una aparición recurrente de cuantificaciones exactas, pero tal como aparece refrendado en la Figura 2, la explicación estilística tiene mejor sustento que la retórica.

Figura 2. Concordance Plot superpuesto

\section{- Cuantificación exacta \\ - Cuantificación difusa}



Otra forma de dar sustento a la explicación estilística por sobre la retórica puede apreciarse en las Tablas 6 y 7. Un n-grama es un método de ventanas móviles a través del cual es posible establecer qué patrones o secuencias son recurrentes en un texto. En la Tabla 6, se muestran los 15 n-gramas más recurrentes de secuencias de tres (trigramas), de cuatro (tetragramas) y de cinco elementos (pentagramas). 
Tabla 6: Ejemplos de los primeros 15 n-gramas (se muestran solo trigramas, tetragramas y pentagramas)

\begin{tabular}{|c|c|l|}
\hline r & F & Patrón o n-gram \\
\hline 1 & 15 & exacta exacta exacta \\
\hline 2 & 12 & exacta exacta exacta exacta \\
\hline 3 & 10 & difusa difusa difusa \\
\hline 4 & 10 & exacta exacta exacta exacta exacta \\
\hline 5 & 7 & difusa difusa exacta \\
\hline 6 & 7 & difusa exacta difusa \\
\hline 7 & 7 & exacta difusa difusa \\
\hline 8 & 6 & difusa exacta exacta \\
\hline 9 & 6 & exacta difusa exacta \\
\hline 10 & 6 & exacta exacta difusa \\
\hline 11 & 5 & difusa difusa difusa exacta \\
\hline 12 & 5 & exacta difusa difusa difusa \\
\hline 13 & 4 & difusa difusa difusa difusa \\
\hline 14 & 4 & difusa difusa exacta difusa \\
\hline 15 & 4 & difusa exacta difusa exacta \\
\hline
\end{tabular}

En la Tabla 6 se muestra que las secuencias no combinadas (lo que debilita la explicación estilística) aparecen en los primeros rangos (1-4), es decir, los más frecuentes. Sin embargo, la sumatoria total de secuencias combinadas es mucho mayor que las secuencias con un solo tipo de cuantificación. Esto puede verse de manera más clara en la Tabla 7.

Tabla 7: Tipos de patrones o n-gramas para probar la explicación estilística

\begin{tabular}{|l|c|c|c|c|}
\hline Tipo de cuantificación & Trigramas & Tetragramas & Pentagramas & Totales \\
\hline $\begin{array}{l}\text { Secuencias solo } \\
\text { cuantificación exacta }\end{array}$ & 13 & 12 & 10 & $\mathbf{3 5}$ \\
\hline $\begin{array}{l}\text { Secuencias solo } \\
\text { cuantificación difusa }\end{array}$ & 10 & 4 & 1 & $\mathbf{1 5}$ \\
\hline Secuencias combinadas & 39 & 50 & 48 & $\mathbf{1 3 7}$ \\
\hline Totales & $\mathbf{6 2}$ & $\mathbf{6 6}$ & $\mathbf{5 9}$ & $\mathbf{1 8 7}$ \\
\hline
\end{tabular}

Tal como se aprecia, el número de n-gramas de secuencias en las cuales se combinan recursos de cuantificación exacta con recursos de cuantificación difusa son los más recurrentes. 
La naturaleza estilística de la cuantificación ya había sido advertida por Espeland y Stevens (1998) en su propuesta para una sociología de la cuantificación. Según estos autores, como cualquier forma expresiva de acción, la cuantificación tiene un importante aspecto estético, ya sea para mostrar números individuales, modelos matemáticos de procesos causales o representaciones gráficas o tabulares de datos numéricos de todo tipo ( $\mathrm{p}$. 422). De acuerdo con Espeland y Stevens (1998), no solo se busca que las cuantificaciones estén libres de errores, sino que sean transmitidas de manera "atractiva, elegante y hermosa" (p. 422). Dado que la mayoría de los ejemplos proporcionados por estos autores refieren a una estética visual (gráficos y figuras), nuestros hallazgos, basados en evidencia lingüística, permiten complementar con lo que podríamos denominar una "estética gramatical".

\section{CONCLUSIONES}

Del análisis realizado de todos los recursos de cuantificación de una cuenta pública anual de una Oficina de Transferencia Tecnológica, se pueden extraer las siguientes conclusiones:

i. Casi la mitad de las veces que aparecen elementos cuantificados, se utiliza una cuantificación difusa.

ii. De los tres tipos de cuantificación difusa, los denominados "difuminantes" son los más frecuentes. Desde el punto de vista gramatical, varios de los difuminantes se manifiestan en cuantificadores indefinidos que afectan a elementos generales que no dicen relación directa con la práctica de la transferencia (años, comités, etapas, etc.).

iii. La cuantificación exacta, en cambio, sí hace referencia a entidades específicamente asociadas con la transferencia tecnológica, como patentes, contratos o tecnologías, y la variación sintáctica de estos recursos es muy baja. Todo lo relativo al dinero y al financiamiento nunca se cuantifica de manera exacta.

iv. Existen varios casos especiales que dan cuenta de usos particulares de cuantificación en el género de las cuentas públicas. Por ejemplo, es muy frecuente el uso de cuantificadores porcentuales, los cuales son, en sí, difusos. Cuando un cuantificador porcentual se combina además con un cuantificador difuso (más del 25\%), la difuminación es aún mayor.

v. El análisis detallado de los datos da soporte a la idea de que la 
cuantificación difuminada, más que un recurso retórico que busca ocultar el número real a la audiencia es una estrategia estilística a través de la cual los actores van intercalando los tipos de cuantificación en el discurso. Este intercalado da cuenta de la naturaleza socialmente transformativa de la cuantificación (Espeland \& Stevens 1998, p. 403).

vi. Por último, el análisis sugiere que este intercalado de estrategias de cuantificación es una práctica social que permite a las OTL variar estilísticamente en la semiosis de los significados de capacidad (en términos de la cantidad de tecnologías y acuerdos que gestionan), benevolencia (en términos del impacto positivo que las tecnologías y acuerdos significan para la sociedad) e integridad (en términos del compromiso que tienen con la innovación). En este sentido, la cuantificación, como hemos demostrado, constituye una herramienta semiótica importante para "crear valor" en el ámbito de la transferencia tecnológica.

Los resultados de este trabajo pueden ser de utilidad para los analistas del discurso interesados en el género de las cuentas públicas. Así también, los hallazgos pueden ser del interés de aquellos encargados de producir este tipo de género para que sean conscientes de cómo es posible utilizar de manera estratégica el recurso gramatical de la cuantificación.

\section{REFERENCIAS BIBLIOGRÁFICAS}

Albelda, Marta. 2008. Atenuantes en Chile y en España: distancia o acercamiento. En A. Briz y otros. Cortesía y conversación: de lo escrito a lo oral, pp. 98-113. Valencia: Universitat de València.

Anthony, Laurence. 2019. AntConc (Version 3.5.8) [Computer Software]. Tokyo, Japan: Waseda University.

Berthoin, Ariane; Michael Hutter and David Stark. 2015. Moments of valuation. Exploring sites of dissonance. Oxford, Inglaterra: Oxford University Press.

Channell, Joanna. 1990. Precise and vague quantities in writing on economics. The writing scholar: Studies in Academic Discourse 3 (1): 95-117.

CoAtes, Jennifer. 1983. The Semantics of the Modal Auxiliaries. Beckenham: Croom Helm.

CORFO, Corporación de Fomento de la Producción. 2016. Informe de Transferencia Tecnológica en Chile. Estudio de caracterización de las actividades y resultados de los procesos de transferencia tecnológica desde los centros de conocimiento en Chile. Santiago, Chile: CORFO.

Di Tullio, Ángela. 2010. Manual de gramática del español. Buenos Aires. Waldhuter editores. 
Doolin, Bill. 2003. Narratives of change: Discourse, Technology and Organization. Organization 10 (4): 751-770.

Doganova, L. (2013). Transfer and exploration: two models of science-industry intermediation. Science and Public Policy 40 (1): 442-452.

Dubois, Betty Lou. 1987. Something on the order of around forty to forty-four: imprecise numerical expressions in biomedical slide talks. Language and Society 16 (1): 527-541.

Dunford, Richard and Deborah Jones. 2000. Narrative in strategic change. Human Relations 53 (9): 1207-26.

Espeland, W. N. \& M. L. Stevens. 2008. A sociology of quantification. European Journal of Sociology/Archives Européennes de Sociologie 49 (3): 401-436.

Fairclough, Norman. 2003. Analyzing discourse. Textual Analysis for Social Research. Londres/Nueva York: Routledge.

Heckscher, Charles and Paul Adler. 2007. The firm as a collaborative community. Reconstructing trust in the knowledge economy. Oxford: Oxford University Press.

Hood, Sue. 2004. Appraising Research: Taking a stance in academic writing. Ph.d. Thesis. University of Technology, Sydney.

Hyland, Ken and Feng Jiang. 2016. Change of attitude. A diachronic study of stance. Written communication 33 (3): 251-274.

HyLAND, Ken. 1996. Writing without conviction: hedging in science research articles. Applied Linguistics 17 (4): 433-54.

2000. Hedges, boosters and lexical invisibility: Noticing modifiers in academic texts. Language Awareness 9 (4): 180-197.

2008. Persuasion, Interaction and the construction of knowledge. Representing self and others in research writing. International Journal of English Studies 8 (2): 1-23.

Kempson, Ruth and Annabel CoRmack. 1981. Linguist and Philosophy 4: 259-309. https:// doi.org/10.1007/BF00350141

Labov, William. 1972. Sociolinguistic patterns. Philadelphia: University of Pennsylvania Press.

LAKOFF, GeORge. 1972. Hedges: a study in meaning criteria and the logic of fuzzy concepts. Proceedings of the Chicago Linguistics Society 8: 183-228.

Latour, Bruno. 1991. Technology is society made durable. En J. Law (ed.). A sociology of monsters: Essays on power, technology and domination, pp. 103-131. Londres: Routledge. 2007. Reassembling the social. Oxford: Oxford University Press.

Law, J. 1994. Organization, Narrative and Strategy. En J. Hassard and M. Parker (eds.). Towards a new theory of organizations, pp. 248-268. London: Routledge.

López, María; Juan Mejía y Rodolfo Schmal. 2006. Un acercamiento al concepto de Transferencia tecnológica en las universidades y sus diferentes manifestaciones. Panorama Socioeconómico 24 (32): 70-86.

Martin, Jim and Peter White. 2005. The language of evaluation. Appraisal in English. London: Palgrave Mc Millan.

Matsuda, Ken; Scott Sadowsky y Omar SAbaj. 2012. Índice de Palabras de Contenido (IPC) y Distribución Porcentual de Legomena (DPL) en artículos de investigación en español. Revista Signos 45 (78): 70-82. https://dx.doi.org/10.4067/S0718-09342012000100005

Mayer, Roger; James Davis and David Schoorman. 1995. An integrative model of organizational trust. Academy of Management Review 20: 709-734.

Miller, Peter. 2001. Governing by numbers: Why calculative numbers matter. Social Research 68 (2): 379-396.

Palmer, Frank. 1990. Modality and the English Modals. London: Longman. 
Pina-Stranger, Álvaro and Emmanuel Lazega. 2011. Bringing personalized ties back in: Their added value for biotech entrepreneurs and venture capitalists interorganizational networks. The Sociological Quarterly 52 (1): 268-292.

Pina-Stranger, Álvaro. 2017. A scenario for cleavage and altercation. En F. Muniesa, L. Doganova, H. Ortiz, A. Pina-Stranger, F. Paterson, A. Bourgoin, V. Ehrenstein, A. Juven, D. Pontille, B. Sarç-Lesavre, \& G. Yon (eds.). Capitalization. A cultural Guide, pp. 5366. Paris: Presses de Mines.

Puga, Juana. 1997. La atenuación en el castellano de Chile. Valencia: Tirant lo Blanch.

Rosch, Eleanor. 1975. Cognitive representations of semantic categories. Journal of Experimental Psychology 104 (3): 192-233.

Scollon, Ron and Suzie Scollon. 2003. Discourses in place. Language in the material world. London, England: Routledge.

Sinclair, AmANDA. 1995. The chameleon of accountability: form and discourses. Accounting, Organization and Society 20 (2/3): 219-237.

Soto, Guillermo. 2017. Una manera de decir: sobre el carácter esencialmente pragmático del lenguaje. Discurso de incorporación a la Academia Chilena de la Lengua. Revista Mapocho 82 (2): 276-301.

Talmy, Leonard. 2000. Towards a Cognitive Semantics. Concept Structuring Systems. Cambridge: MIT.

VAn LeEuwen, THeO. 2008. Discourse and practice. New tools for critical discourse analysis. Oxford, England: Oxford University Press.

WodaK, Ruth. 2015. Performing Europe: backstage versus frontstage politics in the European Parliament. En C. Carter and M. Lawn (eds.). Governing Europe's spaces, pp. 27-55. Manchester, England: Manchester University Press. 